\title{
Ross C. Puffer: Neurosurgery primary board review
}

\section{Publishers New York/Stuttgart, 2018, ISBN: 9781626239272 Thieme, 170 pp, 217 illustrations, paperback/softback}

\author{
Basil E. Grüter ${ }^{1} \cdot$ Lazar Tosic $^{1} \cdot$ Martin N. Stienen ${ }^{1}$ (D) \\ Received: 15 October 2019 / Accepted: 21 October 2019 / Published online: 12 November 2019 \\ (C) Springer-Verlag GmbH Austria, part of Springer Nature 2019
}

Ross C. Puffer presents "Neurosurgery Primary Board Review," a contemporary question and answer book of 170 pages (paperback), primarily intended for residents preparing their neurosurgical board examination. The book is divided into seven chapters, according to the structure of the American Board of Neurological Surgery (ABNS) primary written exam, as follows: neurosurgery, neurology, neuroanatomy, neurobiology, neuropathology, neuroimaging, and fundamental skills.

The book's key feature is the abundant and up-to-date question bank, comprising 1500 multidisciplinary questions. Of those, 600 multiple-choice questions can be found in the book, accompanied by corresponding answer keys that include short explanations for each answer. Those questions are also enriched by 217 illustrations, which are suitably placed within the text. The remaining 900 questions can be accessed online.

Besides the comprehensive question bank, Puffer's book stands out for several further reasons: Firstly, in a rapidly evolving field such as modern neurosurgery examination forms and their contents are constantly changing. The author indicates that the questions were composed when preparing for the 2017 ABNS exam. Here, the presented question bank appears upto-date and covers currently relevant topics in sufficient detail and depth. Secondly, we highly appreciated the links for further reading, specifically for every single answer explanation. These links refer to current editions of common neurosurgical textbooks (e.g., Greenberg's "Handbook of Neurosurgery," or Greenstein's "Color Atlas of Neurosurgery”), even indicating

Basil E. Grüter and Lazar Tosic contributed equally to this work.

Martin N. Stienen

mnstienen@gmail.com

1 Department of Neurosurgery, University Hospital Zurich \& Clinical Neuroscience Center, University of Zurich, Frauenklinikstrasse 10, 8091 Zürich, Switzerland the relevant pages of those textbooks. Therefore, Puffer's book may not only be used to prepare for an exam situation but can also be used as a comprehensive guide for reviewing relevant topics. Furthermore, the author succeeds in remaining conciseeven when explaining complex topics - which helps to keep the text blocks reasonably short. Finally, the questions in the book are clearly formulated with a homogeneous language that is easy to understand, also for non-native English speakers.

On the other hand, the compactness of the book pays the price of selectiveness. In particular, the chapters about "neurosurgery" and "fundamental skills" could comprise a larger number of questions that would optimally cover additional topics. Moreover, the emphasis of this book lays very specifically on the ABNS exam. The structures of the written EANS exam (and probably also of other, national written exams in Europe) follow slightly different structures with different weighing of content, which cannot all be addressed in this book's question bank. Ultimately, whereas many answers are very thoroughly explained with further information beyond the topic, to our disappointment, this structure did not pervade the entire book. For instance, in the neuroanatomy chapter often only one simple answer is indicated, and information about the other structures/ answer options (and their anatomical characteristics) is lacking.

Those who have diligently studied using the book can simulate the written ABNS examination, which is included in the online content. The particular value of this 375 -question trial exam lies in optimizing the time performance and testing the individual's fatigue under realistic conditions.

In summary, Puffer's question and answer book may be particularly helpful for residents preparing for a neurosurgical board examination. It comprises many aspects of contemporary relevant topics in neurosurgery and is ideal for self-assessment. For advanced studies and in order to gain a deeper knowledge, it specifically refers to other modern textbooks. We consider this book an ideal companion for focussed board examination studies and knowledge review in the final stage. 